\title{
Correction to: West Highland White Terriers under primary veterinary care in the UK in 2016: demography, mortality and disorders
}

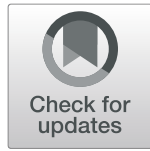

Dan G. O'Neill ${ }^{1 *}$, Zoie F. Ballantyne ${ }^{1}$, Anke Hendricks², David B. Church², Dave C. Brodbelt ${ }^{1}$ and Camilla Pegram ${ }^{1}$

\section{Correction to: Canine Genet Epidemiol (2019) 6:7 \\ https://doi.org/10.1186/s40575-019-0075-2}

In the original publication of this article [1], due to an error in a single count relating to the denominator used for this study, some of the derived values were wrong, so that abstract, plain English summary, results and Fig. 1 all need to be revised.

\section{Abstract}

Update from

Results: WHWTs comprised 6605/905,544 (0.7\%) dogs under veterinary care during 2016 from 886 clinics.

to

Results: WHWTs comprised 6605/336,865 (1.96\%) dogs under veterinary care during 2016 from 438 clinics.

\section{Plain English Summary}

Update from

WHWTs comprised 6605 (0.7\%) of the overall 905,544 study dogs.

to

WHWTs comprised 6605 (1.96\%) of the overall 336, 865 study dogs.

\section{Results}

Demography and mortality

Update from

The study population of 905,544 dogs under veterinary care attending 886 clinics in the vetCompass database during 2016 included 6605 (0.7\%) WHWTs. Annual proportional birth rates showed that WHWTs decreased in popularity from $1.69 \%$ of the annual VetCompass birth cohort in 2004 to $0.43 \%$ in 2015 (Fig. 1).

to

The study population of 336,865 dogs under veterinary care during 2016 at 438 clinics in the VetCompass database included 6605 (1.96\%) WHWTs. Annual proportional birth rates showed that WHWTs decreased in popularity from $4.79 \%$ of the annual VetCompass birth cohort in 2004 to $0.90 \%$ in 2015 (Fig. 1).

\section{Figure 1 Legend}

Update from

Fig. 1 Annual proportional birth rates (2004-2015) for West Highland White Terriers $(n=6605)$ among all dogs $(n=905,544)$ under UK primary veterinary care from January 1st 2016 to December 31st, 2016 at practices participating in the VetCompass ${ }^{\mathrm{TM}}$ Programme

\section{to}

Fig. 1 Annual proportional birth rates (2004-2015) for West Highland White Terriers $(\mathrm{n}=6605)$ among all dogs $(n=336,865)$ under UK primary veterinary care from January 1st 2016 to December 31st, 2016 at practices participating in the VetCompass ${ }^{\text {Tix }}$ Programme

The authors apologize for any confusion this may have caused.

\footnotetext{
Author details

'Pathobiology and Population Science, The Royal Veterinary College, Hawkshead Lane, North Mymms, Hatfield, Herts AL9 7TA, UK. ${ }^{2}$ Clinical Sciences and Services, The Royal Veterinary College, Hawkshead Lane, North Mymms, Hatfield, Herts AL9 7TA, UK.
}

Published online: 28 October 2019

Reference

1. O'Neill DG, et al. West Highland White Terriers under primary veterinary care in the UK in 2016: demography, mortality and disorders. Canine Genet Epidemiol. 2019;6:7.

\footnotetext{
* Correspondence: doneill@rvc.ac.uk

${ }^{1}$ Pathobiology and Population Science, The Royal Veterinary College,

Hawkshead Lane, North Mymms, Hatfield, Herts AL9 7TA, UK

Full list of author information is available at the end of the article
}

(c) The Author(s). 2019 Open Access This article is distributed under the terms of the Creative Commons Attribution 4.0 International License (http://creativecommons.org/licenses/by/4.0/), which permits unrestricted use, distribution, and reproduction in any medium, provided you give appropriate credit to the original author(s) and the source, provide a link to the Creative Commons license, and indicate if changes were made. The Creative Commons Public Domain Dedication waiver (http://creativecommons.org/publicdomain/zero/1.0/) applies to the data made available in this article, unless otherwise stated. 